\title{
Cognitive decline, primary healthcare service use and receipt of informal care within 5 years post-stroke
}

\author{
Daniela Rohde1, Eva Gaynor², Margaret Large ${ }^{3}$, Lisa Mellon¹, Linda Brewer ${ }^{4}$, Patricia \\ Hall $^{3}$, David Williams ${ }^{4}$, Kathleen Bennett ${ }^{1}$, Elizabeth Callaly ${ }^{5}$, Eamon Dolan ${ }^{6}$, Anne Hickey ${ }^{1}$ \\ ${ }^{1}$ Division of Population Health Sciences, Royal College of Surgeons in Ireland, Dublin, Ireland. \\ 2Department of Medicine, Royal College of Surgeons in Ireland, Dublin, Ireland. \\ ${ }^{3}$ Clinical Research Centre, Royal College of Surgeons in Ireland, Beaumont Hospital, Dublin, Ireland. \\ ${ }^{4}$ Department of Geriatric and Stroke Medicine, Royal College of Surgeons and Beaumont Hospital, Dublin, Ireland \\ ${ }^{5}$ Mater Misericordiae University Hospital, Geriatric Medicine, Dublin, Ireland. \\ ${ }^{6}$ Connolly Hospital Blanchardstown, Geriatric Medicine, Dublin, Ireland.
}

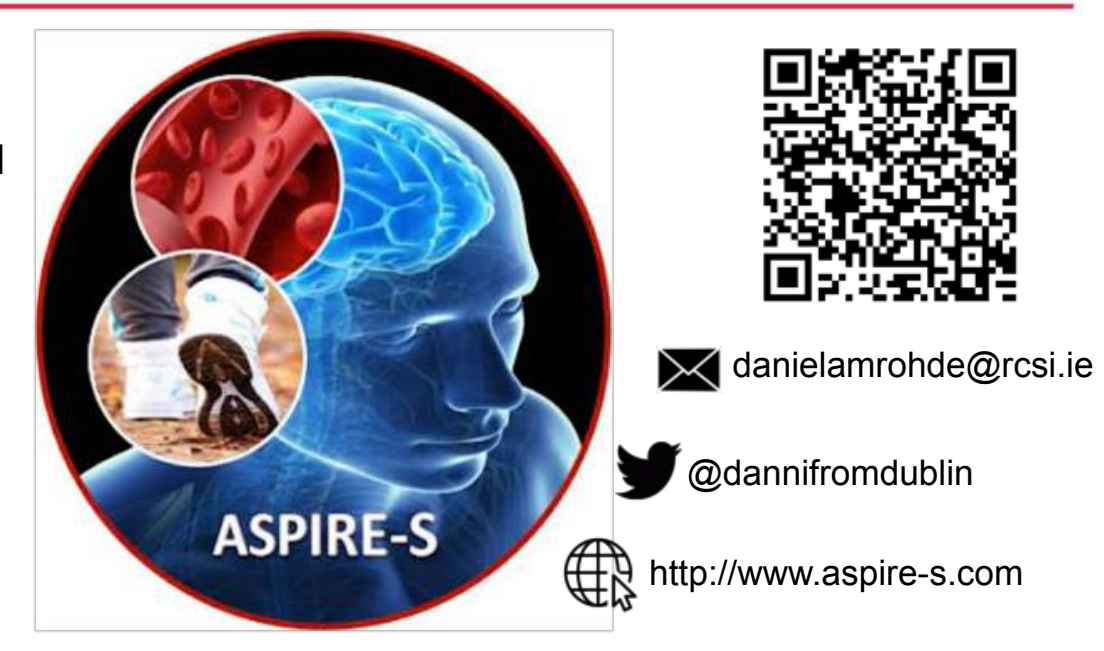

\section{Background}

Stroke is a leading cause of disability worldwide. ${ }^{1}$ Cognitive impairment is common post-stroke, is associated with poorer outcomes, and can increase levels of dependency, leading to a greater burden on carers and the healthcare system. ${ }^{2}$ A number of studies of community-dwelling older adults have indicated that cognitive impairment is associated with greater use of healthcare services. ${ }^{3,4}$ Similarly, cognitive impairment in stroke patients has been reported to be associated with increased costs of care utilisation in the first year poststroke. ${ }^{5}$ However, it is not known to what extent decline in cognitive function impacts on health service use in the longer term post-stroke.

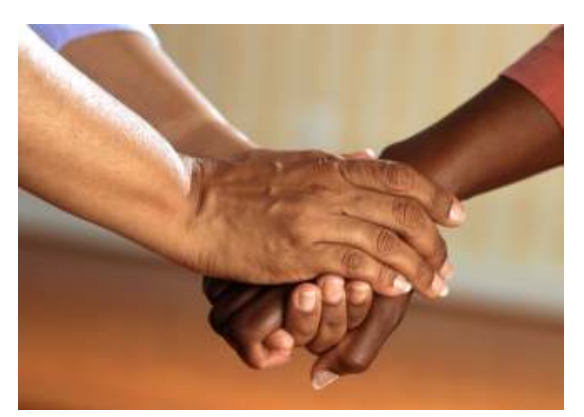

\section{Aim}

The aim of this study was to profile cognitive decline within 5 years post-stroke, and to explore associations with primary healthcare service use and receipt of informal care.

\section{Methods}

Patients from the Action on Secondary Prevention Interventions and Rehabilitation in Stroke (ASPIRE-S) cohort were followed up at 6 months and 5 years post-stroke (Figure 1). ${ }^{1,6}$ Cognitive impairment was assessed using the Montreal Cognitive Assessment (MoCA). Cognitive decline was defined as a decline of $\geq 2$ points in MoCA scores, which has previously been reported to be associated with cognitive decline according to a formal neuropsychological test battery. ${ }^{7}$ Primary healthcare service use at five years post-stroke was defined as the number of self-reported general practitioner visits in the previous 12 months ( $\leq 4$ visits vs. $\geq 5$ visits). Informal care was defined as any unpaid care received by stroke patients from family members, neighbours or friends.
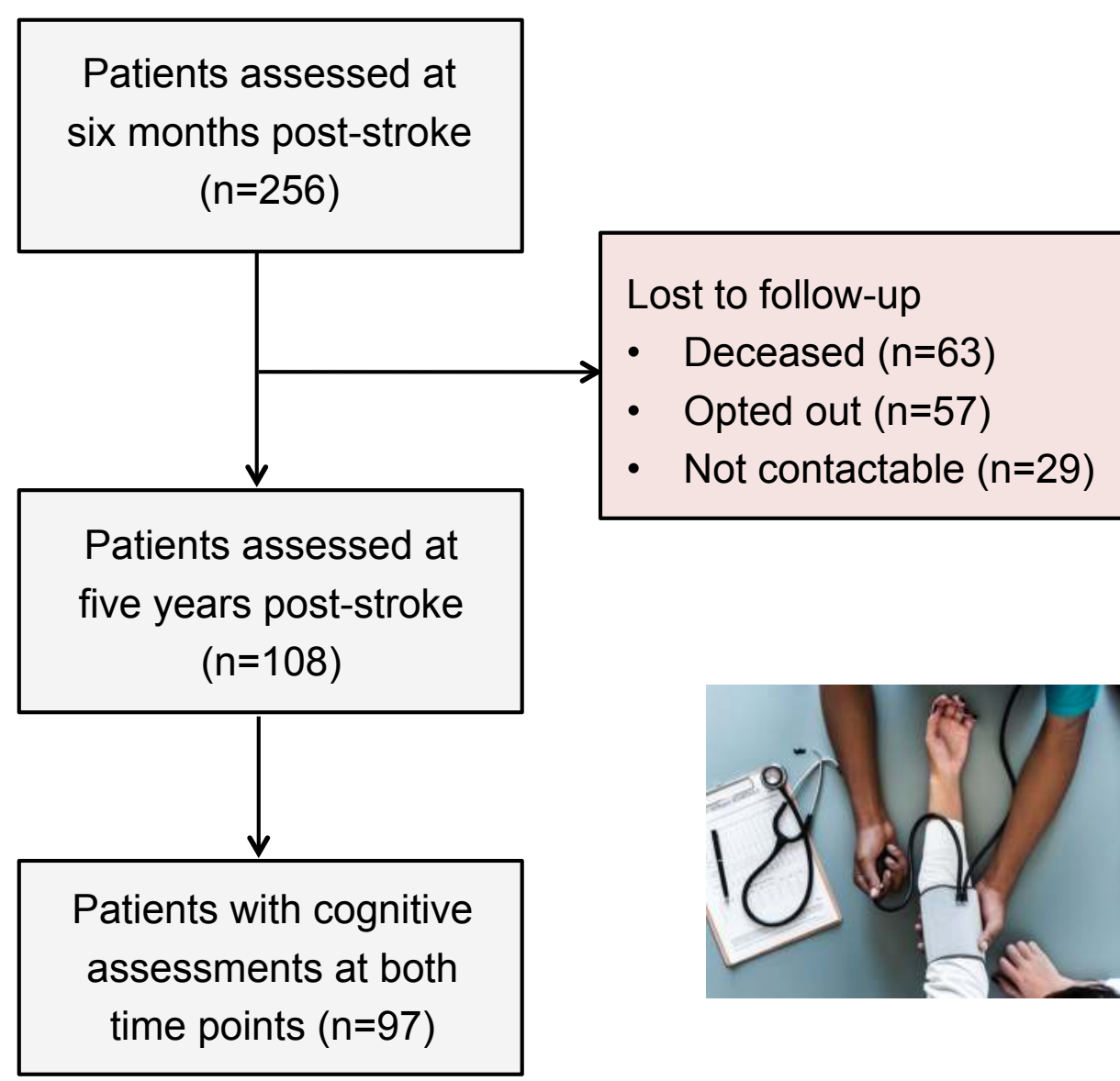

Figure 1. Flow chart of ASPIRE-S participants

\section{Results}

Ninety-seven patients had cognitive assessment at both 6 months and five years post-stroke. Eighteen patients (18.6\%) experienced some improvement in cognitive function, while $16(16.5 \%)$ had either no change in MoCA scores, or a decline of 1 point. Cognitive decline was evident in $63(65.0 \%)$ patients at 5 years (Figure 2).

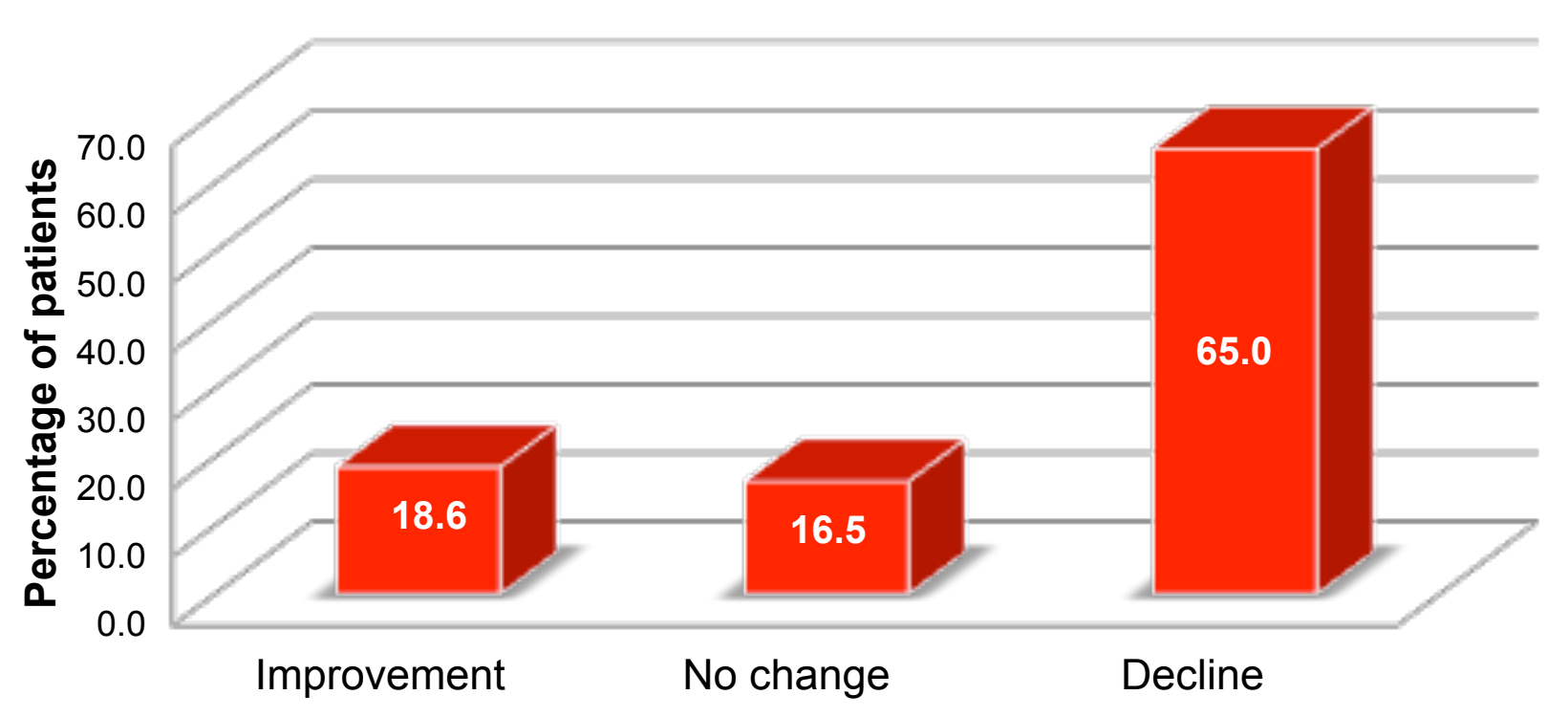

Change in cognitive function from six months to five years

Figure 2. Prevalence of cognitive decline within five years post-stroke

Controlling for age and stroke severity, cognitive decline was associated with a greater number of GP visits in the previous 12 months [OR $(95 \% \mathrm{Cl})): 6.10$ $(1.52,24.44)]$, but not with likelihood of receiving informal care [OR $(95 \% \mathrm{Cl})$ : $1.73(0.43,7.03)]$ (Table 1$)$.

Table 1. Adjusted ORs $(95 \% \mathrm{Cl})$ for logistic regression models of primary healthcare service use and receipt of informal care at five years post-stroke, based on cognitive decline

\begin{tabular}{|c|c|c|}
\hline & Primary health service use & Receipt of informal care \\
\hline & \multicolumn{2}{|c|}{ aOR $(95 \% \mathrm{CI})$} \\
\hline Cognitive decline & $6.10(1.52,24.44)^{*}$ & $1.73(0.43,7.03)$ \\
\hline Model & $\begin{array}{r}\quad X^{2}(3)=14.78, p=.002, \\
\text { pseudo } R^{2}=0.131, n=97\end{array}$ & $\begin{array}{r}X^{2}(3)=41.12, p<.001, \\
\text { pseudo } R^{2}=0.358, n=97\end{array}$ \\
\hline
\end{tabular}

Note: Models adjusted for age and stroke severity. ${ }^{*} p<.05$

\section{Conclusion}

Cognitive decline is evident in a significant number of patients at five years poststroke, and is associated with increased use of primary healthcare services. Further research is required to explore predictors and outcomes of cognitive decline post-stroke, and to investigate cognitive rehabilitation options for patients.

\footnotetext{
References

Feigin VL, Forouzanfar MH, Krishnamurthi R, et al. Global and regional burden of stroke during 1990-2010: findings from the Global Burden of Disease Study 2010. Lancet. 2014;383(9913):245-54

Mellon L, Brewer L, Hall P, et al. Cognitive impairment six months after ischaemic stroke: A profile from the ASPIRE-S study. BMC Neurology. 2015;15(1)

Aigbogun MS, Stellhorn R, Krasa H, et al. Severity of memory impairment in the elderly: Association with health care resource use and functional limitations in the United States. Alzheimers Dement (Amst). 2017;8:51-9. Andrews JS, Desai U, Kirson NY, et al. Functional limitations and health care resource utilization for individuals with cognitive impairment without dementia: Findings from a United States population-based survey. Alzheimers Demen (Amst). 2017;6:65-74

Claesson L, Linden T, Skoog I, et al. Cognitive impairment after stroke - impact on activities of daily living and costs of care The

Rohde D. Williams D, Gaynor $E$, et al. Secondary prevention and cognitive function after stroke: a study protocol for a 5 year follow-up of the ASPIRE-S cohort. BMJ Open. 2017:7(3):e014819.

Tan $\mathrm{HH}, \mathrm{X}, \mathrm{J}$, Teoh HL, et al. Decline in changing Montreal Cognitive Assessment (MoCA) scores is associated with poststroke cognitive decline determined by a formal neuropsychological evaluation. PLoS One. 2017;12(3):e0173291.
} 